\title{
Magnetic resonance imaging sequences for prostate cancer triage: two is a couple, three is a crowd?
}

\author{
Piet Dirix ${ }^{1,2}$, Siska Van Bruwaene ${ }^{3}$, Hendrik Vandeursen ${ }^{4}$, Filip Deckers ${ }^{5}$ \\ ${ }^{1}$ Department of Radiation Oncology, Iridium Kankernetwerk, Oosterveldlaan 22, 2610 Wilrijk (Antwerp), Belgium; ${ }^{2}$ Translational Cancer Research \\ Unit (TCRU), Centre for Oncological Research (CORE), University of Antwerp, Edegem (Antwerp), Belgium; ${ }^{3}$ Department of Urology, AZ \\ Groeninge Hospital, President Kennedylaan 4, 8500 Kortrijk, Belgium; ${ }^{4}$ Department of Urology, ${ }^{5}$ Department of Radiology, GZA Sint-Augustinus \\ Hospital, Oosterveldlaan 24, Wilrijk (Antwerp), Belgium \\ Correspondence to: Piet Dirix. Department of Radiation Oncology, Iridium Kankernetwerk, Oosterveldlaan 22, 2610 Wilrijk (Antwerp), Belgium. \\ Email piet.dirix@gza.be. \\ Provenance: This is an invited article commissioned by Section Editor Xiao Li (Department of Urology, Jiangsu Cancer Hospital \& Jiangsu Institute \\ of Cancer Research \& Nanjing Medical University Affiliated Cancer Hospital, Nanjing, China). \\ Comment on: Jambor I, Verho J, Ettala O, et al. Validation of IMPROD biparametric MRI in men with clinically suspected prostate cancer: A \\ prospective multi-institutional trial. PLoS Med 2019;16:e1002813.
}

Submitted Sep 11, 2019. Accepted for publication Sep 18, 2019.

doi: $10.21037 /$ tau. 2019.09 .42

View this article at: http://dx.doi.org/10.21037/tau.2019.09.42

\section{Background}

Prostate cancer (PCa) can exhibit a wide spectrum of clinical behavior, ranging from extremely indolent to highly lethal. For clinicians, the main challenge is to avoid overdiagnosis and consequently over-treatment of incidental tumors while at the same time not underestimating any clinically significant prostate cancers (SPCa). The standard diagnostic pathway in men who present with an elevated serum prostate specific antigen (PSA) consisted of transrectal ultrasound (TRUS)-guided biopsies with systematic "template" sampling of the entire prostate gland without prior knowledge of the likely locations of the tumor(s). As a result, many men without cancer underwent unnecessary biopsies, clinically insignificant cancers were too often detected, and clinically significant cancers sometimes missed.

It was therefore expected that the use of more innovative imaging as a triage test before prostate biopsy could reduce unnecessary biopsies as well as over-diagnosis of clinically insignificant prostate cancer and would improve detection of clinically significant cancer. In that respect, diagnostic prostate magnetic resonance imaging (MRI) examinations have gained wide-spread acceptance over the last decade. When T2-weighted images are combined with functional, usually diffusion-weighted (DW) and dynamic contrast- enhanced (DCE), sequences in so-called multi-parametric (mp) MRI, information is provided on not only tissue anatomy but also on tissue characteristics such as prostate volume, cellularity, and vascularity. Consequently, mpMRI allows to detect the site(s) of major cancer burden against a background of normal prostate tissue or low-grade, clinically irrelevant disease. Clearly, successful implementation of this technique is heavily dependent on high-quality image acquisition, interpretation, and report communication, all areas addressed by the Prostate Imaging-Reporting and Data System (PI-RADS) recommendations (1).

The seminal PROMIS trial conclusively introduced mpMRI into the diagnostic pathway of prostate cancer in 2017 (2). It convincingly showed that conventional "template" biopsies missed up to $37 \%$ of SPCa (defined as Gleason $\geq 3+4$ or a maximum cancer core length $\geq 6 \mathrm{~mm}$ ). Use of mpMRI as a triage test might have allowed $27 \%$ of men with elevated PSA to avoid an unnecessary biopsy and would have prevented the diagnosis of $5 \%$ fewer clinically insignificant cancers. If subsequent TRUS-guided biopsies were directed by mpMRI findings, up to $18 \%$ more cases of clinically significant cancer might have been detected compared with the standard pathway.

The second pivotal trial establishing the validity of the MRI pathway was the PRECISION trial, published in 
2018 (3). In that study, 500 men with elevated PSA were randomized between standard "template" versus MRIguided biopsies. In the MRI group, men underwent a targeted biopsy (without standard biopsy cores) only if the mpMRI was suggestive of prostate cancer; men whose mpMRI results were not suggestive of prostate cancer were not offered biopsy. The MRI pathway spared $28 \%$ of men from undergoing biopsies and detected $38 \%$ of SPCa, comparing favorable to the conventional pathway arm where everyone received biopsies and only $26 \%$ of patients were diagnosed with SPCa $(\mathrm{P}=0.005)$. Additionally, fewer men were diagnosed with clinically insignificant cancer in the MRI pathway.

Recently, two prospective trials investigated whether the addition of systematic "template" biopsies to all patients in the MRI pathway is still necessary. In the French MRIfirst study, detection of SPCa was significantly lower with systematic TRUS biopsy only (15\%) than with targeted biopsy only (20\%), making the value of the latter beyond discussion (4). When strictly using the aforementioned MRI pathway, $32.3 \%$ of men were diagnosed with SPCa. Adding systematic biopsies in all (including the $21 \%$ with negative MRI) would increase the detection of SPCa to $37.5 \%$. This small but potentially relevant improvement comes at the cost of increasing the detection of clinically insignificant PCa from $6 \%$ to $22 \%$ of the study population. The Dutch $4 \mathrm{M}$ trial had similar results, increasing the detection of SPCa cancer from $25.4 \%$ to $30.4 \%$ when adding systematic biopsies in all men (including the $48 \%$ with negative MRI). Detection of clinically insignificant PCa would in consequence increase from $14 \%$ to $23 \%$ (5).

Finally, the prospective FUTURE trial added some important information to the choice of technique for targeted biopsies (6). In that study, cognitive MRI-guided TRUS biopsy was compared to MRI-TRUS fusion biopsy as well as in-bore MRI-targeted techniques in men with prior negative biopsies but persistent high suspicion of PCa. No significant differences in the detection rates of clinically significant prostate cancer among the three MRI-based targeted biopsy techniques were observed.

Recently, a Cochrane meta-analysis was performed, confirming that the MRI pathway has the most favorable outcome in significant and insignificant PCa detection compared with systematic biopsy (7). Omitting systematic biopsy in men with negative MRI or as an addition to targeted biopsy in men with a positive MRI might be considered acceptable in some clinical situations. The most recent edition of the EAU guidelines now also recommend performing mpMRI before a first set of biopsies (8). The guidelines still recommend the combination of systematic and targeted biopsies in biopsy-naïve men with suspect MRI but provide possibilities for avoiding the systematic biopsies in other situations. But the place of mpMRI as a triage test before biopsies is now firmly established.

\section{The MULTI-IMPROD trial}

It is important to note that most, if not all, abovementioned trials included DCE sequences in their mpMRI protocols. However, PI-RADS version 2 (PI-RADS v2) and its 2019 update v2.1 relegated dynamic contrast enhanced imaging to a minor clarification role secondary to $\mathrm{T} 2$ and DW $(1,9)$. Essentially, it can only upgrade an equivocal finding (score 3 ) on diffusion-weighting in the peripheral zone into a score $4(1,9)$. Therefore, it is estimated that less than $20 \%$ of men need DCE to generate the PI-RADS version 2 score, raising the exciting possibility of limiting the initial screening prostate MRI to a shorter, non-contrast exam $(10,11)$.

The researchers from Turku University have developed such a rapid biparametric (bp) MRI (bpMRI) protocol, that takes only 15 minutes to acquire images and is executed without intravenous contrast agent. Interestingly, DW is performed in 3 separate acquisitions to maximize the contrast between normal tissues and cancer, and differentiate cancer from susceptibility artifacts, which can decrease the diagnostic performance of diffusion-weighted imaging. This concept was first validated in the singleinstitution prospective IMPROD (Improved Prostate Cancer Diagnosis-Combination of Magnetic Resonance Imaging and Biomarkers) trial and demonstrated $90 \%$ negative predictive value (NPV) for SPCa (12).

Subsequently, the authors initiated a larger, prospective, multicenter trial (MULTI-IMPROD, NCT02241122). These results were recently published in PLoS Medicine (13). Between February 1, 2015, and March 31, 2017, 364 men with a clinical suspicion of PCa (i.e., two repeated PSA measurements ranging from 2.5 to $20.0 \mu \mathrm{g} / \mathrm{L}$ and/or an abnormal digital rectal examination) were enrolled at 4 different institutions in Finland. The primary outcome of the trial was diagnostic accuracy, including overall accuracy, sensitivity, specificity, NPV, and positive predictive value, of IMPROD bpMRI for SPCa, which was defined as a Gleason score $\geq 3+4$. Each man with at least one IMPROD score 3-5 lesion received first MRI-guided targeted biopsies followed by a 12 -core "template" performed by the same 
operator, who was aware of the MRI results. For men without suspect lesions on bpMRI, only "template" biopsies were performed.

The results were again quite promising. In men without suspect lesion(s) on bpMRI, the majority of the biopsies yielded no cancer and only $5 \%$ of men harbored SPCa. Overall, bpMRI achieved a NPV of $95 \%$ and $97 \%$ sensitivity, suggesting an excellent potential as triage test. It is clear that adding bpMRI to the standard pathway would reduce unnecessary biopsies as well as over-diagnosis of clinically insignificant prostate cancer, without missing a relevant number of significant cancers. No statistically significant differences in (clinically significant) cancer detection rates were observed between targeted and "template" biopsies.

These results correspond to the recently published BICOD trial, using a very simple bpMRI protocol consisting of axial T2-weighted imaging and single DW acquisition but also taking PSA density (PSAd) into account (14). In BICOD, 808 biopsy-naïve men with clinical suspicion of localized PCa (PSA $<20 \mathrm{ng} / \mathrm{mL}$ and digital rectal examination not suspicious of extra-capsular extension or seminal vesical invasion) underwent upfront bpMRI followed by standard and targeted biopsies of any suspicious bpMRI findings. Significant prostate cancers were detected in $283 / 808$ (35\%) men. The best strategy was restricting biopsies to men with highly suspicious bpMRI findings or PSAd $\geq 0.15 \mathrm{ng} / \mathrm{mL} / \mathrm{cc}$. This reduced the number of men requiring biopsies by $41 \%(329 / 808)$ and overdiagnosis of insignificant cancers by $45 \%$ (79/177), while missing only $5 \%(17 / 329)$ of men with SPCa.

Clearly, these trials are not a comparison between mpMRI (i.e., with DCE) vs. bpMRI. In IMPROD, only 4 (3\%) of all 146 significant prostate cancers were missed. Whether any of those 4 cancers would have been detected with DCE sequences is a moot point. Obviously, the actual number of SPCa is unknown (negative "template" biopsies do not necessarily exclude SPCa), so this percentage could still be an underestimation. It is reassuring that all men in the trial undergo close follow-up, since this will allow to estimate SPCa missed on both bpMRI and "template" biopsies. Still, the overall accuracy of bpMRI (70\%) compares favorably to both PROMIS and PRECISION trials.

\section{Conclusions}

From a clinical point of view, the availability of available
mpMRI slots hampers its wide-spread use as a triage test in men with clinical suspicion of PCa. Clearly, reducing its acquisition time from approximately 30 minutes to about 15 minutes, and leaving aside the hassle of endorectal coil and intravenous contrast, potentially doubles the number of available slots. It is already well established that DCE adds little to the interpretation of diagnostic prostate MRI. To put it succinctly, DWI is considered as the "dominant" sequence in the peripheral zone, as is T2WI in the transition zone. Therefore, many institutions, including our own, have already dropped the DCE sequences from their diagnostic prostate MRI protocols. Clearly, the increased opportunity of offering an MRI, albeit without DCE, as a triage test before biopsies vastly outweighs the very small chance of missing a possible SPCa that would have been detected on mpMRI. Thanks to prospective trials like (MULTI-)IMPROD and BICOD, it is now apparent that "multi" can be safely reduced to "bi"-parametric MRI for the triage of suspected prostate cancer.

\section{Acknowledgments}

None.

\section{Footnote}

Conflicts of Interest: The authors have no conflicts of interest to declare.

Ethical Statement: The authors are accountable for all aspects of the work in ensuring that questions related to the accuracy or integrity of any part of the work are appropriately investigated and resolved.

\section{References}

1. Weinreb JC, Barentsz JO, Choyke PL, et al. PI-RADS prostate imaging - reporting and data system: 2015, version 2. Eur Urol 2016;69:16-40.

2. Ahmed HU, Bosaily AE, Brown LC, et al. Diagnostic accuracy of multi-parametric MRI and TRUS biopsy in prostate cancer (PROMIS): a paired validating confirmatory study. Lancet 2017;389:815-22.

3. Kasivisvanathan V, Rannikko AS, Borghi M, et al. MRItargeted or standard biopsy for prostate-cancer diagnosis. N Engl J Med 2018;378:1767-77.

4. Rouvière $\mathrm{O}$, Puech $\mathrm{P}$, Renard-Penna R, et al. Use of prostate systematic and targeted biopsy on the basis of 
multiparametric MRI in biopsy-naive patients (MRIFIRST): a prospective, multicentre, paired diagnostic study. Lancet Oncol 2019;20:100-9.

5. Van der Leest M, Cornel E, Israe B, et al. Head-to-head comparison of transrectal ultrasound-guided prostate biopsy versus multiparametric prostate resonance imaging with subsequent magnetic resonance-guided biopsy in biopsy-naïve men with elevated prostate-specific antigen: a large prospective multicenter clinical study. Eur Urol 2019;75:570-8.

6. Wegelin O, Exterkate L, van der Leest M, et al. The FUTURE trial: a multicenter randomised controlled trial on target biopsy techniques based on magnetic resonance imaging in the diagnosis of prostate cancer in patients with prior negative biopsies. Eur Urol 2019;75:582-90.

7. Drost FH, Osses DF, Nieboer D, et al. Prostate MRI, with or without MRI-targeted biopsy, and systematic biopsy for detecting prostate cancer. Cochrane Database Syst Rev 2019;4:CD012663.

8. Mottet N, van den Bergh RCN, Briers E, et al. EAUESTRO-ESUR-SIOG guidelines on prostate cancer. Arnhem, The Netherlands: European Association of Urology; 2019. Available online: https://uroweb.org/ guideline/prostate-cancer/

9. Turkbey B, Rosenkrantz AB, Haider MA, et al. Prostate imaging reporting and data system version 2.1: 2019

Cite this article as: Dirix P, Van Bruwaene S, Vandeursen H, Deckers F. Magnetic resonance imaging sequences for prostate cancer triage: two is a couple, three is a crowd? Transl Androl Urol 2019;8(Suppl 5):S476-S479. doi: 10.21037/tau.2019.09.42 update of prostate imaging reporting and data system version 2. Eur Urol 2019;76:340-51.

10. De Visschere P, Lumen N, Ost P, et al. Dynamic contrastenhanced imaging has limited added value over T2weighted imaging and diffusion-weighted imaging when using PI-RADSv2 for diagnosis of clinically significant prostate cancer in patients with elevated PSA. Clin Radiol 2017;72:23-32.

11. Roh AT, Fan RE, Sonn GA, et al. How often is the dynamic contrast enhanced score needed in PI-RADS version 2? Curr Probl Diagn Radiol 2019. [Epub ahead of print].

12. Jambor I, Boström PJ, Taimen P, et al. Novel biparametric MRI and targeted biopsy improves risk stratification in men with a clinical suspicion of prostate cancer (IMPROD Trial). J Magn Reson Imaging 2017;46:1089-95.

13. Jambor I, Verho J, Ettala O, et al. Validation of IMPROD biparametric MRI in men with clinically suspected prostate cancer: a prospective mult-institutional trial. PLoS Med 2019:16:e1002813.

14. Boesen L, Nørgaard N, Løgager V, et al. Prebiopsy biparametric magnetic resonance imaging combined with prostate-specific antigen density in detecting and ruling out Gleason 7-10 prostate cancer in biopsy-naïve men. Eur Urol Oncol 2019;2:311-9. 